\title{
ARTÍCULOS
}

\section{ASOCIACIONISMO INMIGRANTE Y NUEVAS FORMAS DE ACCIÓN POLÍTICA. FACEBOOK Y LA PARTICIPACIÓN POLÍTICA ONLINE DE INMIGRANTES MEXICANOS EN ESTADOS UNIDOS.}

\author{
Leticia Hernández Vega \\ Universidad de Guadalajara \\ vega2907@hotmail.com \\ Luis Escala Rabadán \\ El Colegio de la Frontera Norte \\ luiser@colef.mx
}

\begin{abstract}
Resumen: Si bien el despliegue de acciones políticas ha sido una de las diversas funciones de las asociaciones de inmigrantes, en tiempos recientes muchos de estos grupos han incursionado en el uso de herramientas digitales para hacer política en favor de sus miembros y connacionales frente a los gobiernos de los países de llegada y de origen. En este artículo documentamos la creciente presencia de las asociaciones de inmigrantes mexicanos en Estados Unidos en las plataformas de Internet y explicamos su importancia al permitir un contacto directo, horizontal e inmediato entre dichas asociaciones, sus miembros y otros actores sociales y políticos, tanto de México como de Estados Unidos. Para ello, analizamos las acciones políticas de diversas asociaciones de inmigrantes mexicanos radicados en dicho país en la plataforma Facebook, con el fin de evidenciar los alcances de esta participación en lo que denominamos la "arena política glocaline". Concluimos que el uso de espacios digitales amplía las dimensiones de la esfera pública, al abrir el acceso a múltiples voces desde la multi localidad, aunque en términos de efectos políticos directos los resultados pueden ser contingentes.
\end{abstract}

Palabras clave: Asociaciones de inmigrantes, participación política, medios digitales, Facebook, inmigrantes mexicanos en Estados Unidos.

Tittle: IMMIGRANT ASSOCIATIONISM AND NEW FORMS OF POLITICAL ACTION. FACEBOOK AND THE ONLINE POLITICAL PARTICIPATION OF MEXICAN IMMIGRANTS IN THE UNITED STATES.

Abstract: Although the deployment of political actions has been one of the various functions of immigrant associations, in recent times, many of these groups have been increasingly using digital tools to make politics in favor of their members and fellow compatriots vis-à-vis the governments. of the countries of arrival and origin. In this article we document the growing presence of Mexican immigrant associations in the United States on Internet platforms, and we explain their importance by facilitating direct, horizontal and immediate contact between associations, their memberships, and other social and political actors, both from Mexico and the United States. For this purpose, we analyze the political actions of various associations of Mexican immigrants based on the Facebook platform, in order to demonstrate the scope of this participation in what we call the "glocaline political arena". We

Recibido: 29-04-2021

Aceptado: 20-05-2021

Cómo citar este artículo: HERNÁNDEZ VEGA, Leticia y ESCALA RABADÁN, Luis. Asociacionismo inmigrante y nuevas formas de acción política. Facebook y la participación online de inmigrantes mexicanos en Estados Unidos. Naveg@mérica. Revista electrónica editada por la Asociación Española de Americanistas [en línea]. 2021, n. 27. Disponible en: $<$ http://revistas.um.es/navegamerica>. [Consulta: Fecha de consulta]. ISSN 1989-211X. 
conclude that the use of digital spaces broadens the dimensions of the public sphere, by opening access to multiple voices from multiple locations. However, their direct political effects may be contingent.

Keywords: Immigrant associations, political participation, digital media, Facebook, Mexican immigrants in the United States.

\section{Introducción}

En décadas recientes hemos podido advertir la creciente presencia de imágenes negativas de las y los migrantes en diversas latitudes del planeta gracias a los sistemas de comunicación global. Esto se explica en buena medida a partir del creciente ascenso de fuerzas políticas que en distintos países han encontrado en el tema migratorio una fuente perfecta para el impulso de agendas y sentimientos nativistas y que han calado entre sus habitantes. Un resultado tangible ha sido el aumento de políticas restrictivas en varias naciones, dirigidas hacia la población migrante que ingresa, transita o vive en ellos, así como el aumento de la percepción negativa entre los ciudadanos hacia dicha presencia. En contraste, hay otros sectores en las sociedades de llegada que defienden a la población migrante, a quienes presentan como víctimas de diversas circunstancias, lo que se deriva en la búsqueda de apoyos para su causa.

No obstante, estas posiciones no contribuyen mucho a la comprensión de la complejidad creciente de los procesos migratorios contemporáneos. Esto se debe a que ambas posiciones, muy extendidas entre los sectores conservadores y liberales del espectro político, parten de suponer la condición migrante como la de sujetos atomizados que se expanden de manera desordenada, o bien como víctimas eternas de las circunstancias que los rodean en sus sociedades de destino o de tránsito. Semejantes ópticas no alcanzan a apreciar las diversas capacidades que los propios inmigrantes despliegan frente a los nuevos entornos tecnologizados en donde se sitúan.

Dentro de los procesos migratorios de la era moderna, uno de los flujos con mayor historia, cantidad y densidad es el de los inmigrantes mexicanos en Estados Unidos. Gracias a esta movilidad que se ha desarrollado por más de un siglo se explica la formación, consolidación y ampliación de redes sociales humanas entre ambos países, vinculando regiones enteras entre lugares de origen y de llegada. El surgimiento y desarrollo de dichas redes ilustra precisamente la acción colectiva de dichos inmigrantes, junto con los efectos que se generan en los lugares de salida y de destino. Este denso entramado de conexiones transnacionales, poco atendido en las esferas políticas y mediáticas, es parte de la denominada "sociedad civil migrante", cuyas formas asociativas han sido su piedra angular por muchos años.

Como ha sido el caso con otros colectivos extranjeros en Estados Unidos, las formas asociativas impulsadas por los inmigrantes mexicanos tienen una historia tan larga como su presencia en múltiples regiones de dicho país, adoptando una extensa variedad dentro de sus comunidades, desde modestas asociaciones deportivas o religiosas, hasta asociaciones cívicas más formales. En esta extensa 
gama de formas asociativas, las agrupaciones basadas en el paisanaje, es decir, la procedencia de un mismo pueblo o región de origen, son probablemente las más numerosas y extendidas. A pesar de contar con una larga historia -cuya existencia era conocida solamente por sus comunidades de inmigrantes- su visibilidad comenzó a aumentar durante los años noventa del siglo XX, dada la labor filantrópica que desarrollaban a favor de sus pueblos en México, así como por su participación en programas de cooperación con el gobierno mexicano para la promoción del desarrollo a nivel local (lo que se llegó a conocer como codesarrollo).

Con el paso del tiempo, estas formas asociativas se han consolidado en términos organizativos, lo que las ha llevado a agruparse en federaciones y confederaciones basadas en los estados o regiones de México. Esto, a su vez, las ha convertido en actores sociales cada vez más importantes, tanto en México, como, en menor medida, en Estados Unidos. De hecho, a partir de la creciente importancia de las políticas de atención y vinculación con sus comunidades en el exterior por parte del gobierno mexicano, estas formas asociativas se convirtieron en piezas centrales de dichas políticas, lo que significó una mayor capacidad de interlocución con los distintos niveles del gobierno.

Asimismo, esto ha conducido también a una ampliación en los espacios de participación de dichos grupos, más allá de los vínculos que establecían con sus pueblos de origen y sus paisanos en Estados Unidos, ya que si bien las relaciones con los distintos niveles del gobierno de México se encaminaban a la promoción del bienestar de sus pueblos de origen, dicha relación solía ser a través de canales convencionales y variaba en términos de su cercanía o autonomía. Sin embargo, en años recientes, estas asociaciones de inmigrantes han incursionado paulatinamente en el uso de los medios digitales dentro de la esfera política con el fin de promover la intervención de los actores políticos en favor de sus miembros y connacionales. Dicha incursión hoy se refleja en el uso y apropiación de las Plataformas Digitales de Interacción en Línea como Facebook, que han permitido que estos grupos logren una incidencia significativa en los distintos ámbitos de acción política tanto del origen como del destino.

En este artículo documentamos la participación política de estas asociaciones de migrantes a través de las Plataformas Digitales de Interacción en Línea como Facebook, y explicamos la importancia del uso y apropiación de estos nuevos recursos digitales para el ejercicio de acciones políticas. Para ello, comenzamos presentando un recuento sobre la trayectoria de sus formas asociativas y posteriormente analizamos las acciones políticas de varias de estas asociaciones dentro de la plataforma Facebook, con el propósito de evaluar los alcances dentro de la "Arena Política Glocaline", es decir, un espacio emergente que muestra la confluencia de la acción política en los planos global, local y digital ${ }^{1}$. Finalmente, reflexionamos sobre los logros en materia política de esta nueva faceta de participación entre dichas agrupaciones.

\footnotetext{
1 Leticia Hernández elabora un desarrollo amplio del concepto. Véase: HERNÁNDEZ, Leticia. La arena política Glocaline. Las organizaciones de migrantes jaliscienses en Estados Unidos y la acción política multi local en Facebook, Guadalajara: Universidad de Guadalajara, 2020.
} 


\section{Redes sociales humanas y asociaciones de inmigrantes mexicanos en Estados Unidos}

Si hay acaso un proceso migratorio que sea por demás emblemático en la historia contemporánea de las migraciones, probablemente el ejemplo más claro sea el de los inmigrantes mexicanos en Estados Unidos. Este flujo cuenta con una historia de al menos cien años, con cambios en su intensidad a lo largo del siglo XX, lo que la convierte en la de mayor densidad y extensión en dicho país de destino, en donde constituye una población de aproximadamente doce millones de personas -tanto documentadas como indocumentadas- lo que las convierte en el mayor colectivo inmigrante en ese país ${ }^{2}$.

Pero, como ocurre con otros procesos migratorios, la llegada y el establecimiento de los inmigrantes mexicanos en Estados Unidos se basa en las redes sociales humanas que fueron construyendo, a lo largo del tiempo, entre sus pueblos de origen y los distintos puntos de llegada en el país de destino. Este proceso ha estado presente a lo largo de la historia de las migraciones. Tal como lo han documentado diversos analistas, la formación de dichas redes comienza a partir de la emigración de uno o varios individuos, y tras su establecimiento en el lugar de llegada, la extensión de la invitación a otros miembros de la familia, a amigos o a paisanos de la misma región o localidad de origen ${ }^{3}$. Estas redes se convierten en un recurso fundamental para facilitar tanto la movilidad entre el lugar de origen y de llegada como para el establecimiento, ya que constituye una fuente para el acceso a información sobre la nueva sociedad, a opciones de empleo y vivienda, e incluso en su acceso a la esfera cívica y política ${ }^{4}$.

Estas redes figuran así como el capital social por excelencia para los emigrantes, lo cual puede ser un elemento fundamental para el logro de algún tipo de movilidad social y económica, así como también para su posible integración en las sociedades de llegada ${ }^{5}$. Las redes sociales humanas también se convierten en

\footnotetext{
${ }^{2}$ ALANÍS, Fernando y ALARCÓN, Rafael (eds.). El ir y venir de los norteños. Historia de la migración mexicana a Estados Unidos (siglos XIX-XXI). México: El Colegio de la Frontera Norte; El Colegio de San Luis; El Colegio de Michoacán, 2016.

${ }^{3}$ BASHI, Vilna. Survival of the Knitted: Immigrant Social Networks in a Stratified World. Stanford: Stanford University Press, 2007; MINES, Richard. Developing a Community Tradition of Migration: A Field Study in Rural Zacatecas, Mexico and California Settlement Areas. La Jolla: Center for U.S.Mexican Studies; University of California San Diego, 1981; MASSEY, Douglas [et al.]. Return to Aztlán. The Social Process of International Migration from Western Mexico. Berkeley: University of California Press, 1987; ROUSE, Roger. Mexican Migration and the Social Space of Postmodernism. La Jolla: Center for U.S.-Mexican Studies; University of California San Diego, 1988; PORTES, Alejandro y RUMBAUT, Rubén. Immigrant America: A Portrait. Berkeley: Univeristy of California Press, 2006.

${ }^{4}$ ALARCÓN-ACOSTA, Rafael. The development of the hometown associations in the United States and the use of social remittances in Mexico. En: DE LA GARZA, Rodolfo y LOWELL, Briant (eds.). Sending Money Home: Hispanic Remittances and Community Development. Nueva York: Rowman \& Littlefield Publishers, 2002, pp. 101-124; DUQUETTE-RURY, Lauren. Collective Remittances and Transnational Coproduction: The $3 \times 1$ Program for Migrants and Household Access to Public Goods in Mexico. Studies in Comparative International Development. 2014, vol. 49, n. 1, pp. 112-139.

5 PORTES, Alejandro. Economic Sociology and the Sociology of Immigration: A Conceptual Overview. En: PORTES, Alejandro (ed.). The Economic Sociology of Immigration. Essays on Networks, Ethnicity, and Entrepreneurship. Nueva York: Russell Sage Foundation, 1995, pp. 1-41; CORNELIUS, Wayne
} 
conexiones entre pueblos y regiones de ambos países, por donde circulan no solamente personas y remesas, sino también mercancías, prácticas sociales e ideas de todo tipo, desde culturales y religiosas hasta políticas. Este extenso tejido de vínculos es la base para poder hablar de circuitos y espacios transnacionales, entre dos o más países ${ }^{6}$.

Las mencionadas redes sociales humanas también son importantes dado que generalmente son el punto de partida para el surgimiento de estructuras asociativas más formales. Dichas asociaciones de inmigrantes emergen en buena medida a partir de alguna necesidad específica en el pueblo de origen, para lo cual sus miembros realizan diversas actividades para la recaudación de fondos. De hecho, ya desde el estudio pionero de Douglas Massey y sus colegas ${ }^{7}$, se enfatizaba la importancia de estas diversas formas asociativas como parte de las redes sociales construidas por los inmigrantes mexicanos en Estados Unidos, lo que ponía de manifiesto su iniciativa colectiva, promoviendo así un sentido de comunidad entre sus integrantes y sus pueblos de origen a lo largo del siglo $\mathrm{XX}^{8}$.

El crecimiento de las asociaciones de inmigrantes mexicanos basadas en el paisanaje llevó a su paulatina agrupación en federaciones, basadas en el estado mexicano en donde se situaban sus pueblos de origen. Este modelo organizativo de asociaciones de pueblo y federaciones se fue desarrollando durante la segunda mitad del siglo XX. Al principio, se trataba de formas auto-organizativas, en la medida en que había escasos contactos con el gobierno mexicano ${ }^{9}$. Sin embargo, estos contactos aumentaron con el paso del tiempo, en buena medida con el despliegue de políticas de acercamiento a su diáspora por parte de los gobiernos de México ${ }^{10}$. De esta forma, las federaciones de inmigrantes mexicanos se fueron

A.; TSUDA, Takeyuki y VALDEZ, Zulema. Human Capital versus Social Capital: A Comparative Analysis of Immigrant Wages and Labor Market Incorporation in Japan and the United States. Migraciones Internacionales. 2003, vol. 2, n. 1, pp. 5-35; LIGHT, Ivan. Deflecting Immigration. Networks, Markets, and Regulation in Los Angeles. Nueva York: Russell Sage Foundation, 2006.

${ }^{6}$ LEVITT, Peggy. The Transnational Villagers. Berkeley: University of California Press, 2001; SMITH, Robert. Mexican New York: Transnational Lives of New Immigrants. Berkeley: University of California Press, 2006; SCHÜTZE, Stephanie. Chicago/ Michoacan: The Construction of Transnational Political Spaces. Latino Studies. 2013, vol. 11, n. 1, pp. 78-102.

${ }^{7}$ MASSEY, Douglas [et al.]. Return to Aztlán... Op. cit.

8 RIVERA-SALGADO, Gaspar y ESCALA-RABADÁN, Luis. Collective Identity and Organizational Strategies of Indigenous and Mestizo Mexican Migrants. En: FOX, Jonathan y RIVERA-SALGADO, Gaspar (eds.). Indigenous Mexican Migrants in the United States. La Jolla: Center for U.S.-Mexican Studies; CCIS-University of California San Diego, 2004, pp. 145-178; FOX, Jonathan y RIVERASALGADO, Gaspar. Building Civil Society Among Indigenous Migrants. En: FOX, Jonathan y RIVERASALGADO, Gaspar (eds). Indigenous Mexican Migrants... Op. cit., pp. 1-65; CRUZ-MANJARREZ, Adriana. Zapotecs on the Move: Cultural, Social, and Political Processes in Transnational Perspective. New Brunswick: Rutgers University Press, 2013; BADA, Xótchil. Mexican Hometown Associations in Chicagoacán: From Local to Transnational Civic Engagement. New Brunswick: Rutgers University Press, 2014.

9 ZABIN, Carol y ESCALA RABADÁN, Luis. From Civic Association to Political Participation: Mexican Hometown Associations and Mexican Immigrant Political Empowerment in Los Angeles. Frontera Norte. 2002, vol. 14, n. 27, pp. 7-41.

10 RIVERA SALGADO, Gaspar. Binational organizations of Mexican migrants in the United States. Social Justice. 1999, vol. 26, n. 3, pp. 27-38; GONZÁLEZ GUTIÉRREZ, Carlos. The Mexican Diaspora in California: The Limits and Possibilities of the Mexican Government. En: LOWENTHAL, Abraham y BURGESS, Katrina (eds.). The California-Mexico Connection. Stanford: Stanford 
convirtiendo en interlocutores importantes de diversas instancias gubernamentales, consolidándose así como el modelo organizativo más visible de sus comunidades inmigrantes en Estados Unidos.

A fines del siglo $\mathrm{XX}$, ante el crecimiento de dichas formas organizativas, el gobierno de México puso en marcha diversos programas enfocados en sus comunidades inmigrantes ubicadas a lo largo y ancho de Estados Unidos. Esto permitió hacer más eficiente la canalización de recursos aportados por dichas comunidades (las llamadas remesas colectivas) y por los distintos niveles gubernamentales, y ciertamente también permitió un incremento en el capital político y una mayor legitimidad del gobierno mexicano ante su enorme diáspora ${ }^{11}$.

Pero una consecuencia adicional de esta nueva relación gobierno-diáspora fue el establecimiento de una creciente participación política por parte de dichas comunidades inmigrantes. Estas formas organizativas se convirtieron en una instancia que permitió a las numerosas comunidades migratorias mexicanas la interacción cada vez más frecuente con los distintos niveles del gobierno de México, dando lugar a una relación a ratos cómoda y a ratos conflictiva entre ambos actores $^{12}$. Pero tal como lo han concluido diversos analistas, la estructura organizativa -promovida por las comunidades inmigrantes mexicanas en Estados Unidos, pero ampliada por la creciente presencia de las políticas gubernamentales-, terminó de consolidar su modelo organizativo ${ }^{13}$. Esto permitió a las federaciones de inmigrantes mexicanos asumirse como promotores de los intereses de sus comunidades ante diseñadores de políticas y tomadores de decisiones, tanto de Estados Unidos como de México, sobre todo de este último. Asimismo, los actores políticos preferían la interlocución con asociaciones que agruparan y presentaran las demandas de los miembros de sus comunidades. La creciente interacción propiciada por estas asociaciones de inmigrantes derivó en lo que Boruchoff ubica como su "potencial didáctico" en lo que se refiere a su participación política, y con ello "en la promoción de una conciencia y agencia políticas"14. Autoras como Ostergaard-

University Press, 1993, pp. 221-235; GONZÁLEZ GUTIÉRREZ, Carlos. La organización de los inmigrantes mexicanos en Los Ángeles: la lealtad de los oriundos. Revista Mexicana de Política Exterior. 1995, n. 46, pp. 59-101; IMAZ, Cecilia. La nación mexicana transfronteras. Impactos sociopolíticos en México de la emigración a Estados Unidos. México: UNAM, Facultad de Ciencias Políticas y Sociales, 2006; MOCTEZUMA, Miguel. La transnacionalidad de los sujetos. Dimensiones, metodología y prácticas convergentes de los inmigrantes mexicanos en los Estados Unidos. México: Universidad Autónoma de Zacatecas; Miguel Ángel Porrúa, 2011.

11 GONZÁLEZ GUTIÉRREZ, Carlos (coord.). Relaciones Estado-diáspora: aproximaciones desde cuatro continentes. México: Miguel Ángel Porrúa Editor, 2006; FERNÁNDEZ DE CASTRO, Rafael; GARCÍA, Rodolfo y VILA, Ana (coords.). El Programa $3 \times 1$ para migrantes: iprimera política transnacional en México? México: Instituto Tecnológico Autónomo de México; Miguel Ángel Porrúa Editor, 2006.

12 GOLDRING, Luin. The Mexican State and Transmigrant Organizations: Negotiating the Boundaries of Membership and Participation. Latin American Research Review. 2002, n. 37, pp. 55-99; ESCALA RABADÁN, Luis. Asociaciones de inmigrantes mexicanos en los Estados Unidos: Logros y desafíos en tiempos recientes. Desacatos. 2014, n. 46, 52-69; BADA, Xóchitl. Mexican Hometown... Op. cit.

13 GONZÁLEZ GUTIÉRREZ, Carlos (coord.). Relaciones Estado-diáspora... Op. cit.; GOLDRING, Luin. Blurring Borders: Constructing Transnational Community Process of México-U.S. Migration. Research in Community Sociology. 1995, n. 6, pp. 69-104; GOLDRING, Luin. The Mexican State and Transmigrant Organizations... Op. cit., pp. 55-99.

14 BORUCHOFF, Judith. From Public Works to Political Actions: Political Socialization in Guerrero 
Nielsen destacan que las redes y organizaciones de inmigrantes facilitan sus prácticas políticas ${ }^{15}$, sin embargo el reto radica en examinar y determinar "el modo en que las prácticas políticas transnacionales de los migrantes contribuyen a procesos políticos democráticos, tanto en el país de origen como en el de residencia"16.

\section{Participación política inmigrante y redes sociales digitales}

Continuando con los planteamientos previos, una articulación muy similar es la que podemos observar en la participación política de los inmigrantes mexicanos en Estados Unidos. Si bien durante muchos años las formas asociativas tradicionales mantuvieron un bajo perfil en dicho país, con esporádicas comunicaciones con instancias del gobierno estadounidense, esto paulatinamente fue cambiando con la incorporación y uso de las herramientas digitales, al punto que dichas organizaciones se convirtieron en interlocutores públicos de actores políticos, especialmente de su país de origen. Esta participación condujo al establecimiento de canales de comunicación digital permanentes, que supusieron que funcionarios mexicanos, alcaldes e incluso gobernadores de estados tuviesen encuentros con sus comunidades de paisanos en distintas ciudades estadounidenses, cuyos resultados es posible seguir a través de Internet.

Así, ante la emergencia de las redes sociales digitales, su uso en la esfera política ha ido incorporándose a la relación entre asociaciones de inmigrantes mexicanos y las instancias de gobierno, lo que aquí analizamos desde la teoría de redes. En la versión más abstracta de esta teoría (desde los tiempos de Jacob L. Moreno $)^{17}$, estas han sido usadas como una metáfora para describir las interacciones sociales entre grupos de personas. Las estructuras compuestas por nodos y arcos, en donde los primeros se usan para representar a los agentes y los segundos para referirse a cualquier tipo de relación entre ellos, permitió no solamente una visión topográfica de la manera en la que las relaciones tienen lugar, sino incluso su análisis matemático.

No obstante, en la actualidad esta metáfora ha sido extendida al campo de las Plataformas Digitales de Interacción en Línea, en donde los miembros de una comunidad se auto-presentan, establecen relaciones e interactúan con otros miembros dentro de la misma comunidad, compartiendo además contenidos de especial interés para ellos, muchos de los cuales son producidos por ellos mismos. De acuerdo con Ellison y Boyd ${ }^{18}$, una plataforma de red social digital es un sitio disponible en línea que cumple con los siguientes requisitos: 1) contiene perfiles

\footnotetext{
Hometown Organizations. Latino Studies. 2013, vol. 11, n. 1, pp. 66-67.

15 OSTERGAARD-NIELSEN, Eva. La política a través de las fronteras: reflexiones sobre la dimensión transnacional de la participación política de los migrantes. En: ESCRIVÁ, Ángeles; BERMÚDEZ, Anastasia y MORAES, Natalia (eds.). Migración y participación política. Estados, organizaciones y migrantes latinoamericanos en perspectiva local-transnacional. Madrid: Consejo Superior de Investigaciones Científicas, 2009, pp. 17-41.

${ }^{16}$ OSTERGAARD-NIELSEN, Eva. La política a través de las fronteras... Op. cit., p. 31.

17 MORENO, Jacob. Fundaments of Sociometry: An introduction. Sociometry. 1941, vol. 4, n. 1, pp. 15-35.

${ }_{18}$ ELLISON, Nicole y BOYD, Danah. Sociality through social network sites. The Oxford handbook of internet studies. 2013, pp. 151-172.
} 
únicos de sus usuarios o miembros, los cuales son públicos o semi-públicos, con límites bien definidos; 2) los perfiles articulan listas de otros usuarios o miembros de la red con quienes se mantienen relaciones de algún tipo (el nombre de estas relaciones varía de una red a otra, aunque los más populares son "Amigos", "Seguidores", "Fans" o "Colegas"); y 3) la posibilidad de que otros usuarios revisen las listas incluidas en los perfiles, con la facilidad de que otros puedan realizar el establecimiento de nuevas relaciones a partir de estas listas.

Considerando la teoría de redes, en este trabajo examinamos a continuación las acciones políticas que han realizado tres de las organizaciones más importantes de migrantes mexicanos en Estados Unidos en la plataforma Facebook en años recientes ${ }^{19}$ : la Federación de Clubes Zacatecanos del Norte de California (FCZNC); la Federación Duranguense USA INC (FDUSA); y la Federación Jalisco Internacional (FEDJALIN). Nuestro propósito es mostrar no solamente que dichas asociaciones canalizan sus acciones políticas a través de la esfera digital, sino que se sitúan en el campo específico de la llamada Arena Política Glocaline, en la cual tres planos - global, local y en línea- se intersectan, dando forma y sentido a acciones políticas emergentes.

19 Si bien los estudios sobre plataformas digitales como Facebook son relativamente nuevos, existen
trabajos previos que muestran el vínculo entre las TIC's y las comunidades migrantes, como MAO, Y.
y QIAN, Y. Facebook use and acculturation: the case of overseas Chinese professionals in Western
countries. International Journal of Communication. 2015, n. 9, pp. 2467-2486; HERNÁNDEZ, Leticia y
PONCE, Antonio. Blogs y migración: apuntes para la comprensión de una nueva relación en ciernes.
En: HERNÁNDEZ E. y HERNÁNDEZ, Leticia (coords.). Las regiones hoy. Políticas públicas,
desarrollo, innovación y procesos migratorios. Guadalajara: Universidad de Guadalajara, 2009, pp.
227-240; HERNÁNDEZ, Leticia y PONCE, Antonio. La voz de los migrantes mexicanos en Estados
Unidos a través de Internet. Guadalajara: Universidad de Guadalajara, 2014; MELELLA, Cecilia E.
Migración y Tecnologías de la Información y de la Comunicación (TIC). La presencia de los periódicos
de migrantes en Internet y los desafíos del análisis de las redes sociales virtuales. Cuadernos de H
Ideas [en leanea]. 2013, vol. 7, n. 7, pp. 1-18. Disponible en
<https://ri.conicet.gov.ar/bitstream/handle/11336/3477/Ar culo MELELLA

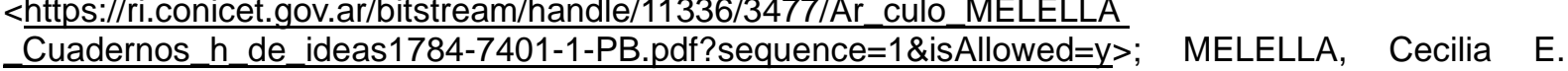
Migraciones transnacionales y uso de las tecnologías de la información y comunicación (TIC). La presencia en Facebook de los periodos de las colectividades de países andinos en la Argentina. Nómadas Revista crítica de Ciencias Sociales y Jurídicas. 2014, vol. 42 n. 2, pp. 1-25; MELELLA, Cecilia E. Migrantes 2.0. Funciones y potencialidades del uso de Internet por las colectividades de migrantes en la Argentina. Trans-pasando Fronteras [en línea]. 2015, n. 7, pp. 61-84. Disponible en $<$ https://doi.org/10.18046/retf.i7.1775>; MELELLA, Cecilia E. Migración y TIC: Identidades andinas en Facebook. La Trama de la comunicación. 2016, vol. 20, n. 1, pp. 73-88; MELELLA, Cecilia E. El uso de las tecnologías de la información y comunicación (TIC) por los migrantes sudamericanos en la Argentina y la conformación de redes sociales transnacionales. Revista Interdisciplinar da Movilidad Humana. 2016, vol. 24 n. 46; LI LI, X. y CHEN, W. Facebook or Renren? A comparative study of social networking site use and social capital among Chinese international students in the United States. Computers in Human Behavior. 2014, n. 35, pp. 116-123. Resulta pertinente anotar que los aportes de este tipo de estudios permiten registrar el proceso bajo el cual han transitado las comunidades de migrantes en tanto agentes de cambio y su vinculación con las tecnologías emergentes. De la misma manera, este tipo de estudios permiten establecer parámetros comparativos en torno a la dimensión de las redes sociales que hoy se extienden del plano humano al plano digital. 


\subsection{Nota metodológica}

Los espacios en Facebook fueron inicialmente analizados con técnicas para grandes cúmulos de datos ${ }^{20}$, con el fin de identificar a todos los participantes de la red $^{21}$. Una vez realizada su identificación, se recabaron las interacciones públicas que sostienen entre sí, para posteriormente ubicarlas en un mapa y organizarlas mediante el algoritmo de Fuchterman y Reingold de 199122. Esta reorganización conllevó cinco pasos: la distribución de los nodos de manera uniforme, la minimización de los cruces de los arcos, el uniformado del largo de estos arcos, el reflejo de la simetría inherente y la conformación de un marco con base en este reflejo. A partir de este análisis se obtuvieron mapas topográficos de las interacciones en cada red que permitieron identificar la manera en la que éstas se concentran en núcleos específicos y la forma en la que se distribuyen a lo largo de todo el espacio, lo que permite tener una noción de la manera en la que los flujos de comunicación en la red son centralizados o no ${ }^{23}$.

Posteriormente, se analizó el contenido de los mensajes publicados por los integrantes de las redes empleadas en este estudio. Este análisis conllevó las siguientes acciones: 1) examinar todas las publicaciones, comentarios y reacciones emocionales (que en Facebook se presentan en forma de "Likes" o "Me gusta", "Me encanta", "Me divierte", "Me entristece", "Me asombra" y "Me enoja"), desde el inicio de actividades en la red hasta el mes de agosto del 2020, para lo cual fueron registradas con fecha, hora y remitente. 2) cada uno de estos contenidos fue revisado y analizado, empleando como referencia la Matriz bidimensional de convergencia propuesta por Hernández (2020), que estructura el ejercicio de acciones políticas multilocales en seis escenarios distintos y se presentan como una caracterización de las formas emergentes de hacer política en línea, tal como se muestra en la siguiente tabla ${ }^{24}$.

20 También conocido como Big Data, por su nombre en inglés, es un conjunto de herramientas y técnicas que permiten el análisis de grandes cantidades de datos generados por los usuarios, principalmente texto, video, imágenes y audio. Se incluyen aquí algoritmos para la adquisición, almacenamiento, recuperación, proceso, análisis, exploración y transformación de datos a gran escala, con la finalidad de obtener información útil a partir de su gestión. LABRINIDIS, Alexandro, JAGADISH, H.V. Challenges and Opportunities with Big Data. Proceedings of the VLDB Endowments. 2012, vol. 5, n. 12, pp. 2032-2033. Cuando se habla de datos a gran escala, se hace referencia a miles de registros, cientos de miles, millones o incluso cantidades mayores.

${ }^{21}$ Cabe aclarar que para ser considerado participante en una red no es necesario estar registrado como "amigo" o como "seguidor" de la misma, basta con haber dado un "like" o cualquier otro tipo de reacción, o con haber publicado un comentario.

22 FRUCHTERMAN, Thomas y RHEINGOLD, Edward. Graph Drawing by Force-directed Placement. Software: Practice and Experience. 1991, vol. 21 n. 11, pp. 1128-1164.

${ }^{23}$ Este análisis pudo ser realizado solamente con aquellos nodos en las redes definidos como de "acceso público", ya que no es posible acceder a las interrelaciones sostenidas por miembros de la red cuando éstas han sido declaradas como "privadas".

24 HERNÁNDEZ, Leticia. La arena política Glocaline... Op. cit., propone esta matriz como parte de una estructura general de uso de Facebook por parte de las organizaciones de migrantes, donde las figuras y las orientaciones se intersectan otorgando sentido al proceso interactivo digital. 


\begin{tabular}{|c|c|c|c|c|}
\hline \multicolumn{5}{|c|}{ Matriz bidimensional de convergencia } \\
\hline & & \multicolumn{3}{|c|}{ Orientaciones } \\
\hline & & Origen & Destino & Ambos \\
\hline & Persona & $\begin{array}{l}\text { Escenario } 1 \\
\text { Acciones orientadas } \\
\text { hacia el origen, } \\
\text { realizadas a título } \\
\text { personal. }\end{array}$ & $\begin{array}{l}\text { Escenario } 2 \\
\text { Acciones orientadas } \\
\text { hacia el destino, } \\
\text { realizadas a título } \\
\text { personal. }\end{array}$ & $\begin{array}{l}\text { Escenario } 3 \\
\text { Acciones orientadas } \\
\text { hacia ambos (origen- } \\
\text { destino) realizadas a } \\
\text { título personal. }\end{array}$ \\
\hline Figuras & Asociación & $\begin{array}{l}\text { Escenario } 4 \\
\text { Acciones orientadas } \\
\text { hacia el origen, } \\
\text { realizadas a } \\
\text { nombre de las } \\
\text { asociaciones }\end{array}$ & $\begin{array}{l}\text { Escenario } 5 \\
\text { Acciones orientadas } \\
\text { hacia el destino, } \\
\text { realizadas a nombre } \\
\text { de las asociaciones. }\end{array}$ & $\begin{array}{l}\text { Escenario } 6 \\
\text { Acciones orientadas } \\
\text { hacia ambos (origen- } \\
\text { destino) realizadas a } \\
\text { nombre de las } \\
\text { asociaciones. }\end{array}$ \\
\hline
\end{tabular}

Tabla 1: Matriz bidimensional de convergencia. Fuente: HERNÁNDEZ, Leticia. La arena política glocaline. Las organizaciones de migrantes jaliscienses en Estados Unidos y la acción política multilocal en Facebook. Guadalajara: Universidad de Guadalajara, 2020, pp. 250-251.

En esta matriz convergen Figuras que constituyen la fuente que genera el contenido publicado (publicaciones realizadas a título personal, o realizadas a título de la asociación); y Orientaciones que indican la dirección que toma el contenido de la publicación (dirigidas hacia el origen, hacia el destino o hacia ambos). De esta forma, estos escenarios caracterizan las siguientes diez formas emergentes en las que las asociaciones de inmigrantes mexicanos en los Estados Unidos hacen política online: a) dar cuenta de encuentros políticos; b) reconocimiento o rechazo a gobernantes, acciones de gobierno y/o leyes; c) manifestaciones de simpatía o rechazo por alguna persona, grupo o partido político; d) difusión y promoción de la democracia; e) información sobre ciudadanía; f) señalamientos sobre el desempeño de las autoridades; g) promoción de campañas políticas; h) recaudación de fondos; i) llamado a la realización de acciones políticas en línea; y j) apoyo a la realización de acciones políticas fuera de línea ${ }^{25}$.

Cabe señalar que, si bien no todas las interacciones y acciones que se observan al interior de estas redes son de corte político, se puede identificar que tienen la finalidad de hacer del sitio un espacio más propicio para las acciones políticas. Estas buscan, por una parte, el fortalecimiento del sitio en términos del incremento en el número de participantes y del aumento en la frecuencia de visita a este, y, por otra, la consolidación del espacio como una fuente confiable de información.

\subsection{Las redes analizadas}

Como ya se mencionó anteriormente, los sitios de Facebook analizados pertenecen a tres asociaciones de inmigrantes mexicanos radicadas en los Estados Unidos, provenientes de los estados de Zacatecas, Durango y Jalisco. La elección de estas redes para buscar sus acciones políticas en y desde Internet se basó en el hecho de que estas asociaciones corresponden a tres trayectorias de migración distintas, cuyos flujos migratorios hacia los Estados Unidos son de alta y mediana intensidad. Las tres asociaciones están constituidas como federaciones, lo que

\footnotetext{
25 lbídem.
} 
supone que aglutinan a diversos clubes de paisanos de sus estados en México. Así como las asociaciones tienen evoluciones políticas diferentes, sus páginas en Facebook, que fueron creadas entre el 2011 y 2013, cuentan con niveles distintos de consolidación como espacios para la intercomunicación entre sus participantes. Mientras que la red jalisciense FEDJALIN es la asociación con mayor experiencia de las tres redes examinadas, cuenta con nueve años y un número de publicaciones de contenidos en Facebook que puede alcanzar las 25 en un solo día, interactuando en ellas hasta 500 personas; las asociaciones de duranguenses y zacatecanos tienen mucha menor experiencia, con siete años cada una y un número mucho menor de publicaciones (en promedio tres o cuatro al mes, con una participación promedio de entre cuarenta y cincuenta personas) ${ }^{26}$. Sin embargo, a pesar de estas diferencias, en los tres espacios analizados es posible identificar el ejercicio de acciones políticas multi locales. Los datos públicos de estas redes fueron recolectados y analizados directamente de sus sitios web, desde la primera publicación hasta agosto de 2020 (tabla 2).

\begin{tabular}{|c|c|c|c|c|}
\hline \multicolumn{5}{|c|}{ Datos generales de las asociaciones } \\
\hline Asociación & Creación & Seguidores & $\begin{array}{c}\text { Reacciones } \\
\text { emocionales } \\
\text { al sitio }\end{array}$ & Autodescripción del sitio \\
\hline $\begin{array}{l}\text { Federación } \\
\text { Duranguense } \\
\text { USA }^{27} \text {. }\end{array}$ & 19/10/2013 & 443 & 429 & $\begin{array}{l}\text { "Es una Organización No } \\
\text { lucrativa, inició operaciones } \\
\text { en abril del 2005 en la } \\
\text { ciudad de Lynwood, Ca, con } \\
\text { el Nombre de "CASA } \\
\text { DURANGO". Fue Fundada } \\
\text { por Don Samuel F. Magaña, } \\
\text { Don Leonardo López, } \\
\text { Agustín Tovar, Carlos } \\
\text { Martínez". }\end{array}$ \\
\hline $\begin{array}{ll}\text { Federación } & \text { de } \\
\text { Clubes } & \\
\text { Zacatecanos } & \text { del } \\
\text { Norte } & \text { de } \\
\text { California }^{28} & \end{array}$ & 09/01/2013 & 2,267 & 2,116 & $\begin{array}{l}\text { "Organización sin fines de } \\
\text { lucro. Nuestro objetivo } \\
\text { principal es organizar a la } \\
\text { gente originaria del estado } \\
\text { mexicano de Zacatecas que } \\
\text { reside en el Norte de } \\
\text { California". }\end{array}$ \\
\hline $\begin{array}{l}\text { Federación } \\
\text { Jalisco } \\
\text { Internacional }^{29} \text {. }\end{array}$ & $28 / 06 / 2011$ & 982,552 & 928,175 & Sin dato \\
\hline
\end{tabular}

Tabla 2: Datos generales de las asociaciones. Fuente: Elaboración propia ${ }^{30}$.

\footnotetext{
${ }^{26}$ Cabe señalar que las federaciones de inmigrantes zacatecanos cuentan con una larga tradición de revistas impresas publicadas, como experiencia previa al uso de Facebook, las cuales fungían como un medio habitual para hacer política.

${ }_{27}$ Disponible en <https://www.facebook.com/Federacion-Duranguense-USA-INC-531654633577447> .

28 Disponible en <https://www.facebook.com/Federacion-de-Clubes-Zacatecanos-del-Norte-deCalifornia-1320289469604>.

${ }^{29}$ Disponible en <https://www.facebook.com/FEDJALIN $>$.

30 Nota: Datos al 16 de agosto del 2020.
} 


\subsection{Topología de las interacciones entre los participantes de las redes}

En términos descriptivos, las topologías obtenidas permiten identificar cómo las tres redes integran un núcleo claramente definido de usuarios que son los que mantienen el mayor tráfico de interacción. Asimismo, se pueden localizar núcleos menores de atracción, periféricos, que concentran las interacciones con cierto número de integrantes y funcionan como medio de enlace con el núcleo, ya que los integrantes en la periferia sostienen un nivel mínimo de interacción con los integrantes del núcleo central.

El siguiente esquema sintetiza gráficamente estas redes y sus componentes. La red de la Federación Duranguense USA INC (FDUSA) presenta un menor número de participantes, donde se identifica un núcleo y seis polos periféricos. Para el caso de la Federación de Clubes Zacatecanos del Norte de California (FCZNC), se pueden identificar el núcleo principal y 17 núcleos periféricos, entre los que sobresale uno, con el mayor nivel de actividad. Finalmente, el mapa de interacciones de la Federación Jalisco Internacional (FEDJALIN) permite identificar dos núcleos centrales, que concentran el mayor nivel de interacción, y múltiples polos periféricos que concentran las interacciones fuera de estos dos núcleos.

a) FDUSA

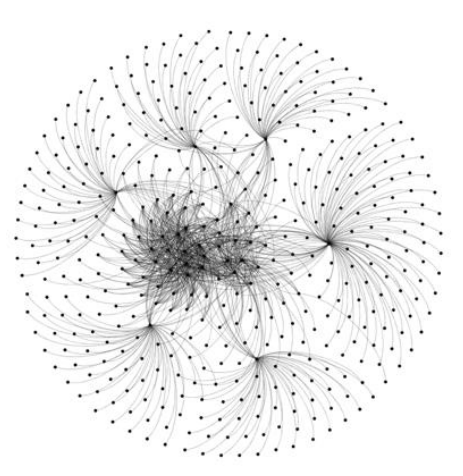

b) FCZNC

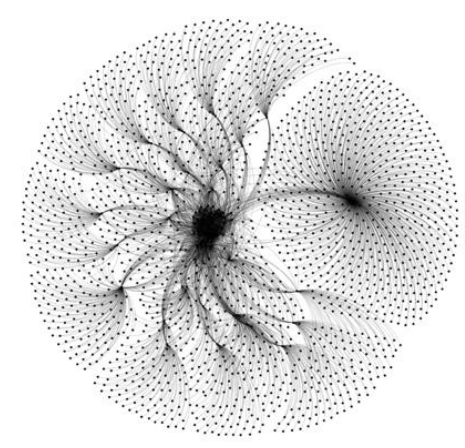

c) FEDJALIN

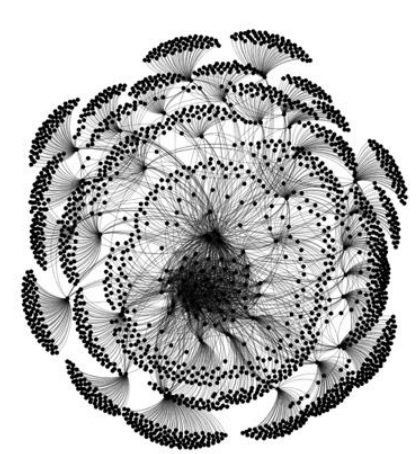

Fig. 1: Flujos de interacción entre los miembros de las redes analizadas. Fuente: Elaboración propia con base en datos obtenidos de Facebook ${ }^{31}$.

Los dos primeros sitios, como se puede ver en la Figura 1, concentran participantes que tienen poca interacción entre sí y cuyo único vínculo es la propia red. En contraste, la tercera red muestra una parte central con participantes con mayor interacción entre ellos, y múltiples polos concentradores con niveles distintos de actividad con el resto de los involucrados. El papel de estos agentes concentradores periféricos se puede ver reflejado en las acciones de orientación política que realizan al interior de la red: tienen mayor número de reacciones emocionales a los mensajes publicados en éstas, constantemente retransmiten las publicaciones hechas en el sitio, o incluso realizan comentarios a nombre de la comunidad $^{32}$. Véase para ello el siguiente ejemplo, en donde uno de los agentes de

\footnotetext{
${ }^{31}$ Nota: Debido a las restricciones actuales de privacidad de Facebook, en este grafo son visibles y se muestran solamente las relaciones de aquellos usuarios que las han declarado como públicas.

32 En el análisis de las interacciones que tienen lugar en Facebook, se contabiliza el número de veces que cada una de estas reacciones es elegida por los usuarios para expresar su emoción ante un
} 
atracción hace un comentario a nombre de la FDUSA:

"Estaremos pendientes de la aprobación, agradecemos la invitacion del Dip[utado] Jesús Ever Mejorado, como de los Dip[utados] Ochoa, Ibarra, Villarreal y el Diputado Benítez, muchas gracias por su mensaje de saludo para los miembros de FDUSA, y para nuestro presidente Don Samuel F Magaña"33.

\subsection{El contenido de las interacciones}

Los espacios en Facebook de las tres federaciones analizadas muestran patrones de comunicación muy similares, aún dadas las diferencias en las dimensiones de las redes conformadas. Se pueden identificar dos grandes tipos de mensajes: en primer lugar, aquellos que buscan consolidar la red en términos del número de participantes y la frecuencia de visita al sitio ${ }^{34}$; y en segundo lugar, aquellos que constituyen el ejercicio de las acciones políticas que las asociaciones realizan en estos espacios.

Con respecto al primer tipo de mensajes, en los primeros años de las redes, las publicaciones buscan asegurar las condiciones para atraer y mantener seguidores apelando a aspectos culturales altamente significativos para sus comunidades, como los llamados a la nostalgia a través de imágenes que evocan el pueblo de origen y la difusión de festividades locales y prácticas sociales que hacen referencia a la identidad comunitaria.

La función de este tipo de mensajes no termina con la consolidación de la red como un espacio de interacción entre los participantes, ya que en los años posteriores, estos se usan para mantener e incluso incrementar el nivel de consolidación alcanzado por la red. Un ejemplo de ello son las publicaciones relacionadas con la difusión y documentación del programa "Corazón de Plata", que tiene por objetivo la reunificación familiar a través de la localización y la facilitación de la intercomunicación entre miembros de familias distantes, tanto en México como en Estados Unidos. Este programa permite que los miembros de las familias no solamente se comuniquen, sino que eventualmente puedan reunirse. Las publicaciones que se relacionan con este programa obtienen reacciones emocionales de los participantes de la red y propician comentarios como los siguientes:

"Gracias a todos los que hicieron este viaje una realidad al poder unir familias con este programa de Corazón de Plata $৩ 0^{\circ}$ ”35.

"Recibimiento en Fremont Ca[lifornia] a los Corazones de Plata que vienen desde Zacatecas a reunirse a sus hijos"36.

Otro medio para consolidar los espacios en Facebook como sitios con alto nivel

mensaje determinado en una unidad de tiempo.

${ }^{33}$ Comentario de un agente atractor a una publicación de la FDUSA del 17 de noviembre del 2016.

${ }^{34}$ La frecuencia de visita a un sitio determinado en Facebook es uno de los indicadores usados para determinar el nivel de popularidad del sitio. Se basa en la medición del número promedio de veces que un usuario accede al sitio en un periodo determinado.

35 Post de FDUSA del 30 de diciembre del 2016.

${ }^{36}$ Post de FCZNC del 26 de agosto de 2018; incluye un video. 
de participación y con una creciente frecuencia de visita, es la publicación de servicios a la comunidad ${ }^{37}$. Los sitios analizados realizan publicaciones que hacen referencia a distintos servicios ofrecidos principalmente para la comunidad de destino, como los siguientes:

"La Federación Duranguense USA ofrece clases de Ciudadanía gratis todos los domingos"38.

"La organización Consejo de Federaciones de Norteamérica (COFEM) llevó a cabo el Taller de Ciudadanía en la Ciudad de Lynwood [California], agradecemos a Anabella Bastida, Francisco Moreno, Norma CASTAÑEDA, Saul Rios, Lety y todos los empleados y voluntarios del COFEM, también a Jesús CASTAÑEDA y su hijo, por el apoyo como a la Abogada de Migracion Érika Vejar Muchas Gracias!!!"39.

"Estimados Amigos Zacatecanos, Este es un recordatorio de que la Feria de Salud en el "Dia del Zacatecano" será este sábado en el Airport Garden Hotel en San Jose, Ca[lifornia]. La Feria de Salud se llevará a cabo de 11:00-03:00 para que traigan a toda su familia y conocidos a esta feria gratuita. Los esperamos!" ${ }^{\prime \prime}$.

Lo anterior es importante porque mostrar solidez organizacional es otro de los elementos que asegura las condiciones para que las acciones políticas tengan mayor alcance. De hecho, en los sitios analizados se encuentran cientos de fotografías que dan cuenta de las diferentes actividades internas, lo cual permite, por un lado, mostrar a sus seguidores que las asociaciones cuentan con una estructura sólida que genera confianza, y por otro, es una manera de rendir cuentas sobre la confianza depositada en ellos. Estas acciones permiten atraer miembros y con ello incrementar el número de participantes, lo cual en el mediano plazo asegura que las acciones políticas implementadas contarán con el eco suficiente para ser difundidas y ejercidas con éxito.

Con respecto al segundo tipo de mensajes, el de las acciones políticas, el análisis de los sitios en cuestión permite apreciar que en los primeros años, las redes parecen estar más orientadas a consolidarse como un sitio de encuentro de los participantes que a la realización de acciones políticas. Es por ello que en los inicios el número de publicaciones de este tipo es muy bajo en relación con el número de publicaciones que buscan la consolidación del sitio. No obstante, una vez que las redes han logrado incrementar el número de participantes, la proporción de publicaciones con acciones políticas se incrementa, aunque no llega a superar el número de las que buscan sostener el nivel de consolidación alcanzado. Ello sugiere que las páginas tratan de asegurar el espacio en el que las acciones políticas puedan realizarse sin correr el riesgo de perder participantes.

En las publicaciones de este tipo se pueden apreciar los vínculos y encuentros que las asociaciones mantienen con funcionarios e instancias gubernamentales del

\footnotetext{
${ }^{37}$ Los niveles de participación se miden a partir los números de mensajes, de respuestas a mensajes publicados, de reacciones emocionales y de retransmisiones de mensajes que tienen lugar en un sitio de Facebook, en un periodo determinado.

38 Post de FDUSA del 19 de febrero de 2018.

39 Post de FDUSA del 25 de marzo de 2017.

40 Post de FCZNC del 29 de mayo de 2014.
} 
origen y el destino en todos los niveles de gobierno, así como con líderes inmigrantes y representantes de las comunidades. Esto supone que los sitios buscan fungir como un lugar de encuentro de la comunidad inmigrante, una ventana al quehacer cotidiano de las asociaciones, vinculada a la rendición de cuentas, y, sobre todo, un espacio ampliado para colocar temas de la agenda política inmigrante conducente a dirimir asuntos públicos. Ello se puede observar en los siguientes posts:

"Este domingo el Gobernador Miguel Alonso Reyes firmó la iniciativa de Ley para la creación de la Secretaría del Zacatecano \#Migrante. Se trata de una iniciativa que surgió de la propia comunidad migrante y que el Titular del Poder Ejecutivo asumió como propia en un afán de fortalecer las relaciones con los miles de zacatecanos que integran los diversos clubes y asociaciones de migrantes en Estados Unidos"41.

"El Club Laguna USA, miembro de esta federación trabajando con la Alcaldesa de Gómez Palacio [Durango], Lic. Leticia Herrrera Ale en el área de energía solar para llevar a la comunidad calentadores de agua" ${ }^{2}$.

"Todos los latinos unidos \#NoDonaldTrump www.facebook.com/FEDJALIN www.facebook.com/ORGULLOSAMENTEJALISCIENSES

www.facebook.com/yosoyjalisco.mx". (Comentario a partir de un video donde un cantante hondureño canta en contra de Donald Trump $)^{43}$.

"Es indignante que a una pobre mujer que lo único que hace el trabajar para sostener su hogar la traten como una criminal, cuántos políticos han robado las arcas de nuestro país, cuánta". (Reacción ante un video donde se muestra abuso por parte de elementos de seguridad de Jalisco) ${ }^{44}$.

"En el marco de México en Los Ángeles el Consulado de México en Los Ángeles y La compañía VOLARIS otorgaron el reconocimiento "HÉROE DE LA COMUNIDAD " a Don Samuel F Magaña presidente de la Federación Duranguense USA INC y a la Sra Rosario Marín Ex Tesorera de los Estados Unidos, en este evento estuvieron presentes altos ejecutivos de VOLARIS, COO JOSE LUIS SUAREZ, CCO HOLGER BLANKENSTEIN, por parte del Consulado de México el EMB. CARLOS GARCÍA DE ALBA, cónsul de comunidades CARLOS GIMÉNEZ, además de líderes comunitarios, medios de comunicación y nuestra amiga la Lic. Malú Yunez de VOLARIS, miembros de esta organización Jesús Campos , Consuelo Campos, Alvaviola García, Stephani Flores, Rafael Abdo, Enedino Aguirre, Jesús Ubaldo Magaña, Sammy Magaña, Diana Magaña, Karina Castelum, Carlos Martínez . En este homenaje Don Samuel F. Magaña, agradeció a los presentes, confirmando la continuidad de seguir apoyando a la comunidad Mexicana, VOLARIS bautizará una aeronave con el nombre de DON SAMUEL F. MAGAÑA"45.

Varios de estos posts ilustran la posición de estas asociaciones con respecto a cuestiones de carácter político, ya sea de apoyo o bien de crítica a instancias

\footnotetext{
${ }^{41}$ Post compartido por la FCZNC, original del Gobierno de Zacatecas del 6 de septiembre del 2014.

42 Post de FDUSA del 4 de noviembre de 2017.

43 Post de FEDJALIN del 5 de marzo de 2016.

44 Post de FEDJALIN del 19 de abril de 2016.

45 Post de FDUSA del 27 de julio de 2017.
} 
gubernamentales. Asimismo, presentan también su capacidad organizativa a través de la realización de eventos que involucran una extensa variedad de actores sociales. Entre los personajes que interactúan en los sitios de Facebook analizados, se encuentran empresarios, políticos y funcionarios de gobierno tanto del origen como del destino, así como otras asociaciones como el Consejo de Federaciones Mexicanas (COFEM), líderes migrantes, comunidad inmigrante, instancias gubernamentales de México, medios de comunicación, y varias empresas, entre otras. En ese sentido, este tipo de anuncios les permite exhibir la diversidad de personajes e instancias que confluyen en estos sitios, lo que a su vez muestra la amplia red de relaciones socio-políticas que puede generar este tipo de agrupaciones, como resultado de sus acciones multilocales.

De acuerdo con los planteamientos señalados por Hernández ${ }^{46}$, citados previamente, se puede apreciar que las asociaciones de inmigrantes mexicanos en Estados Unidos aquí examinadas, realizan acciones políticas multilocales en línea bajo las diez formas emergentes antes señaladas, aunque no con la misma frecuencia e intensidad. Entre las formas más socorridas se encuentran: dar cuenta de encuentros políticos, información sobre ciudadanía, difusión y promoción de la democracia, manifestaciones de simpatía o rechazo por alguna persona, grupo o partido político, reconocimiento o rechazo a gobernantes, acciones de gobierno y/o leyes y recaudación de fondos. Por otro lado, las acciones políticas emergentes menos socorridas (pero no menos importantes) son: señalamientos sobre el desempeño de las autoridades, promoción de campañas políticas, llamado a la realización de acciones políticas en línea, y apoyo a la realización de acciones políticas fuera de línea. Y aunque la frecuencia de estas últimas publicaciones es menor, sí es posible dar cuenta de ellas, dado que son evidentes en más de una ocasión en los diferentes sitios.

A partir del análisis realizado, cabría hacer dos señalamientos importantes. Por un lado, el uso de las plataformas digitales para la realización de acciones políticas por parte de las asociaciones de inmigrantes mexicanos en Estados Unidos muestra que no son necesariamente una extensión de las que se realizan en el plano offline. Esto quiere decir que las organizaciones examinadas mantienen sus agendas, negociaciones y acuerdos con actores políticos. Más bien, se trata de acciones emergentes que se ponen en marcha a partir de la disponibilidad de dichas plataformas. No obstante, como señalamos, esto no significa la desaparición de las acciones políticas convencionales, sino que se adopta una coexistencia de distintas lógicas de hacer política, las cuales se hacen más complejas al confluir en el plano glocaline.

Por otro lado, el uso de las plataformas digitales por parte de estas organizaciones, para comunicarse entre sí y con otros, constituye una transformación importante de la esfera pública, al ampliar considerablemente las posibilidades de interacción no solamente entre ciudadanos, sino que abre la posibilidad de interpelar a las figuras políticas a partir de la participación en grupos sociales que buscan un reconocimiento al sentido de pertenencia y la reivindicación de derechos. En ese sentido, los inmigrantes organizados en agrupaciones como las

\footnotetext{
${ }^{46}$ HERNÁNDEZ, Leticia. La arena política Glocaline... Op. cit., pp. 250-251.
} 
aquí examinadas, a través de sus acciones políticas en espacios online, despliegan lo que podría denominarse una "ciudadanía glocaline", que implica un ejercicio de la ciudadanía a través de las redes sociales virtuales, desde múltiples localidades y a través de colectivos organizados. De esta forma, Internet se convierte en un potencial espacio público de mayor deliberación y participación, donde estos colectivos inmigrantes abordan asuntos públicos con el ánimo de promover el bienestar de sus comunidades en ambos lados de la frontera entre México y Estados Unidos.

\section{Conclusiones}

Por décadas, la labor filantrópica de las asociaciones inmigrantes en favor de sus comunidades, en Estados Unidos, pero sobre todo en sus pueblos de origen, los condujo a interactuar con actores políticos, y con ello, desplegar acciones de distintos tipos. Sin embargo, en tiempos recientes y al igual que otros grupos, dichas asociaciones han incursionado en el uso de Internet para reproducir o ampliar sus acciones políticas. A partir del análisis de la plataforma Facebook, en este trabajo hemos documentado y examinado el uso de las redes digitales en las acciones políticas realizadas por las asociaciones de inmigrantes mexicanos en Estados Unidos, y hemos evidenciado la emergencia de la Arena Política Glocaline como espacio de convergencia de dicha acciones.

Las acciones políticas en línea de estas asociaciones se han convertido en un elemento emergente, no declarado en los objetivos iniciales de las organizaciones, pero ejercido gradualmente conforme la estructura de la red de participantes lo ha ido permitiendo. Por ejemplo, ninguna de las tres páginas de Facebook de las asociaciones de migrantes analizadas se presenta en su auto-descripción como espacios para la acción política, sino que más bien se presentan como espacios para el encuentro entre los inmigrantes y las personas con las que se relacionan, tanto en el lugar de origen como en el destino. Muchos de los participantes de las redes así lo asumen, y de hecho, así se puede apreciar a través de sus comentarios, cuando mandan saludos, preguntan por alguna familia en especial, o informan acerca de algún suceso. Esta finalidad inicial se debe sostener, con el fin de asegurar la presencia de los participantes.

Las páginas de Facebook analizadas de las tres organizaciones elegidas muestran publicaciones que en principio buscan conseguir un mayor número de participantes y una mayor frecuencia de interacciones, pero que han expandido su rango de acción hacia la realización de acciones políticas en la red. La acción política en Facebook requiere de participantes activos, no solamente de seguidores, puesto que los primeros participan frecuentemente revisando los contenidos y reaccionando ante ellos, ya sea mediante sus comentarios, sus reacciones emocionales o su interés por difundirlos (compartir). De esta manera, es posible identificar diversos niveles de actividad en cuanto a las acciones políticas en Facebook, los cuales se incrementan conforme la red va aumentando su número de participantes y la frecuencia con la que éstos consumen los contenidos que la red difunde y participan a propósito de ellos. En esta consolidación de las redes, dos elementos son importantes: las publicaciones que se realizan en la red y los propios miembros de la misma, puesto que facilitan e impulsan a otros usuarios a participar. 
Así, se comienzan a identificar y reforzar cada vez más, diversos polos de concentración que funcionan como enlace con grupos pequeños de participantes, lo que otorga a las redes conformaciones topológicas muy especiales, como se puede apreciar en los grafos previamente presentados.

En este sentido, el uso de plataformas como Facebook por parte de las organizaciones de migrantes muestra un cambio significativo en los procesos comunicativos, extendiendo su capacidad de interlocución con los distintos actores políticos, tanto del origen como del destino, y por ende, los efectos que de esta relación se desprenden suelen ser positivos y permanentes en el tiempo. Al apropiarse de recursos digitales, estas organizaciones contribuyen a la transformación del espacio público de manera colectiva, pues abren la posibilidad a los integrantes de extensas comunidades inmigrantes de comunicarse e interpelar a figuras del ámbito político, desde múltiples localidades, observando dichas transformaciones en el plano glocaline.

Recapitulando, es necesario enfatizar en el hecho de que esta nueva faceta de participación política de las organizaciones de migrantes, a través de medios digitales permite evidenciar la construcción de un incipiente ejercicio de la ciudadanía a través del uso de dichas plataformas digitales. De hecho, este uso está orientado en varias ocasiones hacia la consolidación de alianzas e intereses con las autoridades políticas, particularmente a nivel municipal y estatal en México, ilustrando así la prevalencia de prácticas políticas de más viejo cuño. Con todo, consideramos que el uso de las Plataformas Digitales de Interacción en Línea como Facebook abren una nueva faceta en la vida de las organizaciones inmigrantes, al hacer posible el acceso horizontal a un mucho mayor número de personas y de grupos, localizados en múltiples comunidades tanto del país de llegada como de origen, transformando así el espacio público previamente establecido, a través de las estructuras tradicionales de hacer política, y abriendo de esta manera las posibilidades para una mayor deliberación y participación ahora desde la palestra digital.

\section{Referencias}

ALANÍS, Fernando y ALARCÓN, Rafael (eds.). El ir y venir de los norteños. Historia de la migración mexicana a Estados Unidos (siglos XIX-XXI). México: El Colegio de la Frontera Norte; El Colegio de San Luis; El Colegio de Michoacán, 2016.

ALARCÓN ACOSTA, Rafael; ESCALA RABADÁN, Luis y ODGERS ORTIZ, Olga. Mudando el hogar al norte. Trayectorias de integración de los inmigrantes mexicanos en Los Ángeles. México: El Colegio de la Frontera Norte, 2012.

ALARCÓN-ACOSTA, Rafael. The development of the hometown associations in the United States and the use of social remittances in Mexico. En: DE LA GARZA, Rodolfo y LOWELL, Briant (eds.). Sending Money Home: Hispanic Remittances and Community Development. Nueva York: Rowman \& Littlefield Publishers, 2002, pp. 101-124. 
BADA, Xótchil. Mexican Hometown Associations in Chicagoacán: From Local to Transnational Civic Engagement. New Brunswick: Rutgers University Press, 2014.

BASHI, Vilna. Survival of the Knitted: Immigrant Social Networks in a Stratified World. Stanford: Stanford University Press, 2007.

BORUCHOFF, Judith. From Public Works to Political Actions: Political Socialization in Guerrero Hometown Organizations. Latino Studies. 2013, vol. 11, n. 1, pp. 55-77.

CORNELIUS, Wayne A.; TSUDA, Takeyuki y VALDEZ, Zulema. Human Capital versus Social Capital: A Comparative Analysis of Immigrant Wages and Labor Market Incorporation in Japan and the United States. Migraciones Internacionales. 2003, vol. 2, n. 1, pp. 5-35.

CRUZ-MANJARREZ, Adriana. Zapotecs on the Move: Cultural, Social, and Political Processes in Transnational Perspective. New Brunswick: Rutgers University Press, 2013.

DUQUETTE-RURY, Lauren. Collective Remittances and Transnational Coproduction: The $3 \times 1$ Program for Migrants and Household Access to Public Goods in Mexico. Studies in Comparative International Development. 2014, vol. 49, n. 1, pp. 112-139.

ELLISON, Nicole y BOYD, Danah. Sociality through social network sites. The Oxford handbook of internet studies. 2013, pp. 151-172.

ESCALA RABADÁN, Luis; BADA, Xóchitl y RIVERA-SALGADO, Gaspar. Mexican Migrant Civic and Political Participation in the U.S.: The Case of Hometown Associations in Los Angeles and Chicago. Norteamérica. 2006, vol. 1 n. 2, $127-$ 172.

ESCALA RABADÁN, Luis. Asociaciones de inmigrantes mexicanos en los Estados Unidos: Logros y desafíos en tiempos recientes. Desacatos. 2014, n. 46, 52-69.

Federación de Clubes Zacatecanos del Norte de California. Disponible en $<$ https://www.facebook.com/Federacion-de-Clubes-Zacatecanos-del-Norte-deCalifornia-132028946960467>.

Federación Duranguense USA INC. Disponible en $<$ https://www.facebook.com/Federacion-Duranguense-USA-INC$\underline{531654633577447>\text {. }}$

Federación Jalisco Internacional. Disponible en <https://www.facebook.com/FEDJALIN>.

FERNÁNDEZ DE CASTRO, Rafael; GARCÍA, Rodolfo y VILA, Ana (coords.). El Programa 3x1 para migrantes: ¿primera política transnacional en México? México: Instituto Tecnológico Autónomo de México; Miguel Ángel Porrúa Editor, 
2006.

FOX, Jonathan y RIVERA-SALGADO, Gaspar. Building Civil Society Among Indigenous Migrants. En: FOX, Jonathan y RIVERA -SALGADO, Gaspar (eds). Indigenous Mexican Migrants in the United States. La Jolla: CCIS; Center for USMexican Studies; University of California, San Diego, 2004, pp. 1-65.

FRUCHTERMAN, Thomas y RHEINGOLD, Edward. Graph Drawing by Forcedirected Placement. Software: Practice and Experience. 1991, vol. 21 n. 11, pp. 1128-1164.

GOLDRING, Luin. Blurring Borders: Constructing Transnational Community Process of México-U.S. Migration. Research in Community Sociology. 1995, n. 6, pp. 69104.

GOLDRING, Luin. The Mexican State and Transmigrant Organizations: Negotiating the Boundaries of Membership and Participation. Latin American Research Review. 2002, n. 37, pp. 55-99.

GONZÁLEZ GUTIÉRREZ, Carlos (coord.). Relaciones Estado-diáspora: aproximaciones desde cuatro continentes. México: Miguel Ángel Porrúa Editor, 2006.

GONZÁLEZ GUTIÉRREZ, Carlos. La organización de los inmigrantes mexicanos en Los Ángeles: la lealtad de los oriundos. Revista Mexicana de Política Exterior. 1995, n. 46, pp. 59-101.

GONZÁLEZ GUTIÉRREZ, Carlos. The Mexican Diaspora in California: The Limits and Possibilities of the Mexican Government. En: LOWENTHAL, Abraham y BURGESS, Katrina (eds.). The California-Mexico Connection. Stanford: Stanford University Press, 1993, pp. 221-235.

HERNÁNDEZ, Leticia. La arena política Glocaline. Las organizaciones de migrantes jaliscienses en Estados Unidos y la acción política multi local en Facebook, Guadalajara: Universidad de Guadalajara, 2020.

HERNÁNDEZ, Leticia y PONCE, Antonio. Blogs y migración: apuntes para la comprensión de una nueva relación en ciernes. En: HERNÁNDEZ E. y HERNÁNDEZ, Leticia (coords.). Las regiones hoy. Políticas públicas, desarrollo, innovación y procesos migratorios. Guadalajara: Universidad de Guadalajara, 2009, pp. 227-240.

HERNÁNDEZ, Leticia y PONCE, Antonio. La voz de los migrantes mexicanos en Estados Unidos a través de Internet. Guadalajara: Universidad de Guadalajara, 2014.

IMAZ, Cecilia. La nación mexicana transfronteras. Impactos sociopolíticos en México de la emigración a Estados Unidos. México: UNAM, Facultad de Ciencias 
Políticas y Sociales, 2006.

LABRINIDIS, Alexandro, JAGADISH, H.V. Challenges and Opportunities with Big Data. Proceedings of the VLDB Endowments. 2012, vol. 5, n. 12, pp. 2032-2033.

LEVITT, Peggy. The Transnational Villagers. Berkeley: University of California Press, 2001.

LEVITT, Peggy. Transnationalizing Community Development: The Case of Migration Between Boston and the Dominican Republic. Nonprofit and Voluntary Sector Quarterly. 1997, vol. 26, n. 4, pp. 509-526.

LI, X. y CHEN, W. Facebook or Renren? A comparative study of social networking site use and social capital among Chinese international students in the United States. Computers in Human Behavior. 2014, n. 35, pp. 116-123.

LIGHT, Ivan. Deflecting Immigration. Networks, Markets, and Regulation in Los Angeles. Nueva York: Russell Sage Foundation, 2006.

MAO, Y. y QIAN, Y. Facebook use and acculturation: the case of overseas Chinese professionals in Western countries. International Journal of Communication. 2015, n. 9, pp. 2467-2486.

MASSEY, Douglas [et al.]. Return to Aztlán. The Social Process of International Migration from Western Mexico. Berkeley: University of California Press, 1987.

MELELLA, Cecilia E. Migración y Tecnologías de la Información y de la Comunicación (TIC). La presencia de los periódicos de migrantes en Internet y los desafíos del análisis de las redes sociales virtuales. Cuadernos de $H$ Ideas [en línea]. 2013, vol. 7, n. 7, pp. 1-18. Disponible en $<$ https://ri.conicet.gov.ar/bitstream/handle/11336/3477/Ar culo MELELLA Cuadernos $\mathrm{h}$ de ideas1784-7401-1-PB.pdf? sequence=1\&isAllowed $=\mathrm{y}>$.

MELELLA, Cecilia E. Migraciones transnacionales y uso de las tecnologías de la información y comunicación (TIC). La presencia en Facebook de los periodos de las colectividades de países andinos en la Argentina. Nómadas Revista crítica de Ciencias Sociales y Jurídicas. 2014, vol. 42 n. 2, pp. 1-25.

MELELLA, Cecilia E. Migrantes 2.0. Funciones y potencialidades del uso de Internet por las colectividades de migrantes en la Argentina. Trans-pasando Fronteras [en línea]. 2015, n. 7, pp. 61-84. Disponible en $<$ https://doi.org/10.18046/retf.i7.1775>.

MELELLA, Cecilia E. Migración y TIC: Identidades andinas en Facebook. La Trama de la comunicación. 2016, vol. 20, n. 1, pp. 73-88.

MELELLA, Cecilia E. El uso de las tecnologías de la información y comunicación (TIC) por los migrantes sudamericanos en la Argentina y la conformación de redes sociales transnacionales. Revista Interdisciplinar da Movilidad Humana. 
2016, vol. 24 n. 46.

MINES, Richard. Developing a Community Tradition of Migration: A Field Study in Rural Zacatecas, Mexico and California Settlement Areas. La Jolla: Center for U.S.-Mexican Studies; University of California San Diego, 1981.

MOCTEZUMA, Miguel. La transnacionalidad de los sujetos. Dimensiones, metodología y prácticas convergentes de los inmigrantes mexicanos en los Estados Unidos. México: Universidad Autónoma de Zacatecas; Miguel Ángel Porrúa, 2011.

MORENO, Jacob. Fundaments of Sociometry: An introduction. Sociometry. 1941, vol. 4, n. 1, pp. 15-35.

OSTERGAARD-NIELSEN, Eva. La política a través de las fronteras: reflexiones sobre la dimensión transnacional de la participación política de los migrantes. En: ESCRIVÁ, Ángeles; BERMÚDEZ, Anastasia y MORAES, Natalia (eds.). Migración y participación política. Estados, organizaciones y migrantes latinoamericanos en perspectiva local-transnacional. Madrid: Consejo Superior de Investigaciones Científicas, 2009, pp. 17-41.

PORTES, Alejandro y RUMBAUT, Rubén. Immigrant America: A Portrait. Berkeley: Univeristy of California Press, 2006.

PORTES, Alejandro. Economic Sociology and the Sociology of Immigration: A Conceptual Overview. En: PORTES, Alejandro (ed.). The Economic Sociology of Immigration. Essays on Networks, Ethnicity, and Entrepreneurship. Nueva York: Russell Sage Foundation, 1995, pp. 1-41.

RIVERA SALGADO, Gaspar. Binational organizations of Mexican migrants in the United States. Social Justice. 1999, vol. 26, n. 3, pp. 27-38.

RIVERA-SALGADO, Gaspar y ESCALA-RABADÁN, Luis. Collective Identity and Organizational Strategies of Indigenous and Mestizo Mexican Migrants. En: FOX, Jonathan y RIVERA-SALGADO, Gaspar (eds.). Indigenous Mexican Migrants in the United States. La Jolla: Center for U.S.-Mexican Studies; CCIS-University of California San Diego, 2004, pp. 145-178.

ROUSE, Roger. Mexican Migration and the Social Space of Postmodernism. La Jolla: Center for U.S.-Mexican Studies; University of California San Diego, 1988.

SCHÜTZE, Stephanie. Chicago/ Michoacan: The Construction of Transnational Political Spaces. Latino Studies. 2013, vol. 11, n. 1, pp. 78-102.

SMITH, Robert. Mexican New York: Transnational Lives of New Immigrants. Berkeley: University of California Press, 2006.

ZABIN, Carol y ESCALA RABADÁN, Luis. From Civic Association to Political 
Participation: Mexican Hometown Associations and Mexican Immigrant Political Empowerment in Los Angeles. Frontera Norte. 2002, vol. 14, n. 27, pp. 7-41. 